\title{
Changes in visual sensitivity associated with voluntary saccades ${ }^{1}$
}

DOUGLAS G. PEARCE, ${ }^{2}$ Defence Research Establishment Toronto, Downsview, Ont., Canada, and ERROL PORTER, Department of Industrial Engineering, University of Toronto, Toronto, Canada

Previous studies of saccadic suppression do not rule out the possibility that the phenomenon may be affected by, or produced by, shifts of the Os' criteria for reporting a flash. The present study, using a presumably criterion-free measure $\left(d^{\prime \prime}\right)$, demonstrates a decrease in visual sensitivity beginning as much as $150-200 \mathrm{msec}$ prior to a voluntary saccade. It is suggested that criterion-free measures of visual sensitivity be used in future studies of the saccadic suppression.

The inhibition of vision during voluntary saccadic eye movements has been postulated to account for the fact that the visual scene does not appear blurred when fixation is rapidly changed from one point to another. Although recent experiments (Volkmann, 1962; Latour, 1962, 1966; Zuber \& Stark, 1966; Volkmann, Schick, \& Riggs, 1968) have demonstrated a decrease in detection performance in the temporal vicinity of voluntary saccades, the magnitude of the effect (equivalent to a reduction in target luminance of the order of $0.5 \mathrm{log}$ unit) seems insufficient to account for the phenomenal lack of blurring of the visual environment when voluntary saccades are performed (Volkmann, 1962). Saccadic suppression, the decrease in visual detection performance associated with voluntary saccades, is presumed by some workers (e.g., Latour, 1966; Volkmann et al, 1968) to be a central inhibitory effect which effectively reduces the magnitude of the visual signal prior to the point at which the presence or absence of the signal is judged. On the other hand, Richards (1969) suggests that the effect is due to the

Fig. 1. The stimulus array used in the experiment.

Fig. 2. $P(Y / S+N)$, correct detections, and $P(Y / N)$, false alarms, as a function of time relative to the onset of a voluntary saccade for $O L F$ in a YN experiment. The average number of observations for each $\mathrm{P}(\mathrm{Y} / \mathrm{S}+\mathrm{N})$ data point is approximately 60 . The average number of observations for each $P(Y / N)$ point is approximately 40 . occurrence of retinal shearing during the saccade, causing an amplification of the retinal background from which the signal must be extracted.

One possibility that has not been excluded is that the O's criterion may be involved in saccadic-suppression experiments. It is possible that criterion shifts may attenuate, enhance, or, in fact, produce the phenomenon of saccadic suppression. The experiments presented in this paper yield data concerning changes in both visual sensitivity ( $\left.d^{\prime}\right)$ and the $O^{\prime}$ 's criterion as a function of time relative to the onset of a voluntary saccade.

\section{PROCEDURE}

The $O$ was seated in a dark room, her head steadied by a mouthbite and headrest; her right eye was occluded. The $O$ 's task was to fixate two lights in succession, moving her eye as quickly as possible to one from the other as they were switched on and off. The stimulus array, shown in Fig. 1, was at a distance of 48 in. from the $O$ 's left eye. For $4 \mathrm{sec}$ she fixated a small light, $F_{1}$, which was then extinguished and the second light, $F_{2}$, was turned on. $F_{2}$
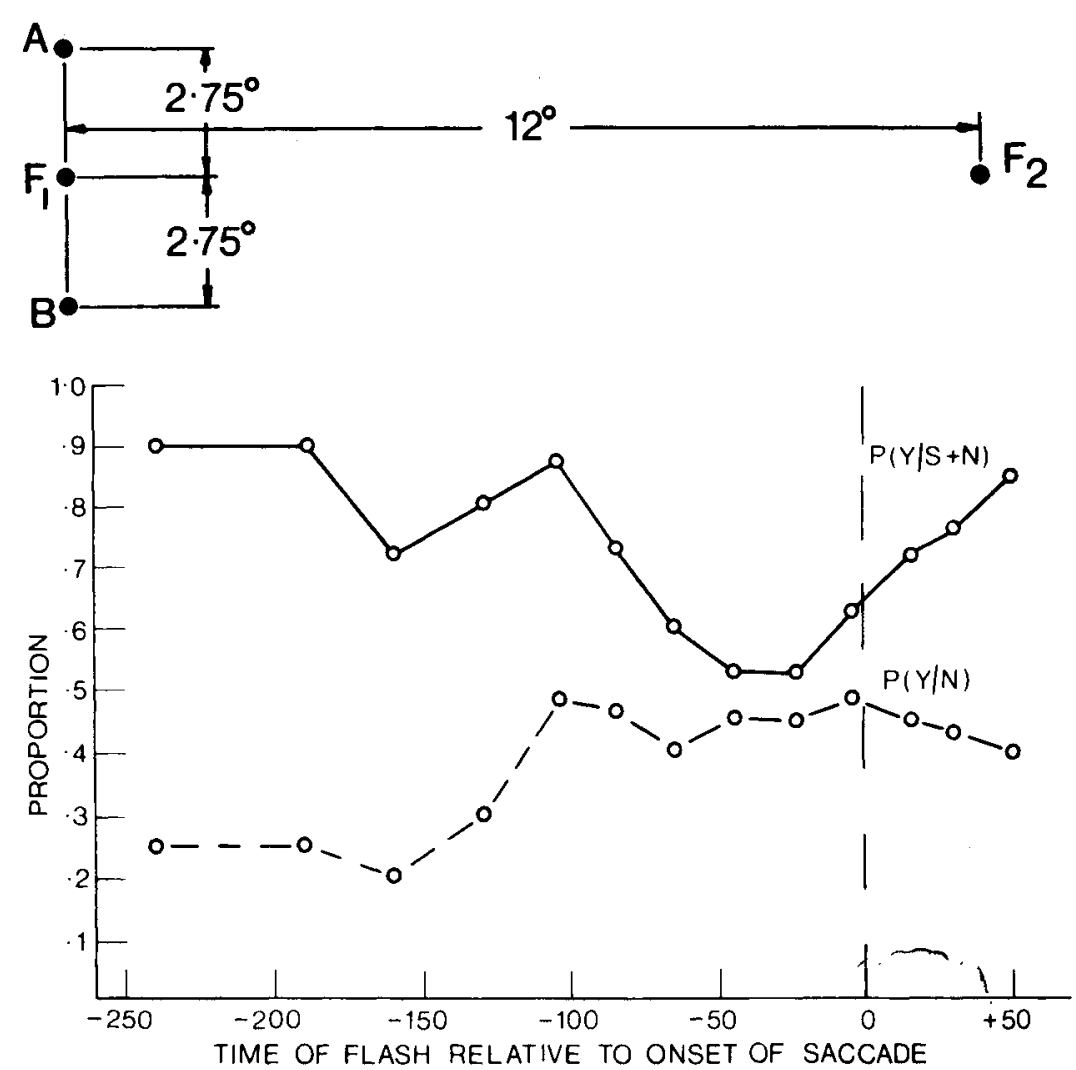

(m sec) was $12 \mathrm{deg}$ of visual angle to the right of $F_{1}$ and in the same horizontal plane. At various times relative to the saccade from $F_{1}$ to $F_{2}$, a test flash occurred at $A$ or $B$, located $2.75 \mathrm{deg}$ directly above and below $F_{1}$, respectively. After $6 \mathrm{sec}, F_{2}$ was extinguished and $F_{1}$ reappeared, beginning the next trial. Both $F_{1}$ and $F_{2}$ were NE-2 neon discharge lamps masked to give circular 0.03-deg targets. The test flashes at $A$ and $B$ each subtended $0.14 \mathrm{deg}$.

Two types of experiments were conducted, yes-no (YN) and two-alternative forced-choice (2AFC). In the $\mathrm{YN}$ experiments, the $\mathrm{O}$ reported whether a flash occurred at A. Correct responses were indicated by a large dim peripheral flash of light. The source at $B$, the cue, flashed with a luminance sufficient to be seen on all trials, while the simultaneous test flash at $\mathrm{A}$ occurred on approximately $60 \%$ of the trials. The luminance of $\mathrm{A}$ was adjusted to yield approximately $90 \%$ correct detections well before the saccade. The luminances of both $A$ and $B$ were adjusted with neutral density filters (Kodak No. 96). The cue flash at B was necessary in order to indicate to the $O$ the instant at which the test flash occurred or, in the case of a blank, would have occurred. The use of this temporal cueing flash enabled us to measure the false-alarm rate, $P(Y / N)$, for the detection of $A$ as a 


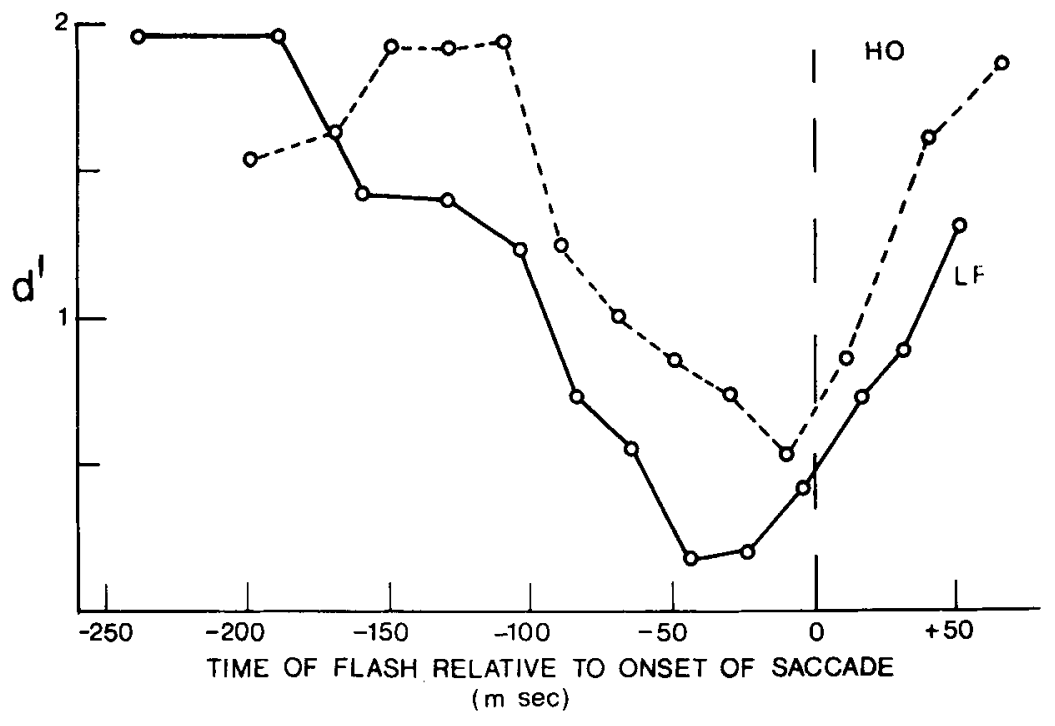

function of time. Without a temporal cue, the false alarms could not apply to any particular time. That B did not have an appreciable masking effect on $A$ was determined in control experiments.

In the 2AFC task, the $\mathrm{O}$ reported whether A or B flashed. In this task, A and $B$ were of equal luminance and adjusted to give approximately $90 \%$ correct responses well before the saccade. The eye-movement task, the timing of the test flashes, and the use of the peripheral flash to give knowledge of results were the same as in the $\mathrm{YN}$ experiments.

Measurement of Saccade Latency

The movements of the left eye in the horizontal plane were recorded using a crude corneal reflection technique. A small rectangle of near-infrared light was focused onto the scleral-iris junction. When the eye moved, the energy incident on a nearby photocell (Hoffman 2A) changed, due to differences in the curvature and reflectance of the portions of the eye passing under the rectangle. The short-circuit current of the photocell was transformed into a voltage by an operational amplifier acting as a current measuring device. With the output of the amplifier adjusted to zero for fixation on $F_{1}$, the output voltage, although clearly nonlinear, was an increasing function of eye rotation to the right toward $F_{2}$. The amplifier fed a Schmitt trigger that was set at a voltage that represented an eye rotation of approximately $0.25 \mathrm{deg}$ to the right. Because of the normal fixation eye movements, the trigger level could not be set to zero. The Schmitt trigger stopped a timer that was started when $F_{1}$ was extinguished, yielding a measure of saccade latency which contained a relatively constant error due to the nonzero trigger level. The constant error in the timer reading was estimated by means of sample chart recordings showing the time $F_{1}$ was extinguished, the time at which the Schmitt trigger changed states, and the eye-movement voltage. These readings were taken on approximately $5 \%$ of the trials. This constant was subtracted from each timer reading in a given session to give the saccade latencies. In the present experiment, the saccades had a duration of the order of $50 \mathrm{msec}$ and a mean latency of the order of $300 \mathrm{msec}$.

Test Flashes and Timing

Sources A and B were Sylvania R1131C glow modulators. Although these tubes were not designed to provide flashes of 10 microsec, a few were found, by testing a large number, which flashed fairly reliably for this duration. It was necessary to maintain a small current through the tubes at all times, however, in order to ensure reliable flashing behavior (Matin, 1964; Engel \& Howat, 1965). The light resulting from this current was not visible to the 0 . Although the luminance of the flashes was not measured, the relative luminance was monitored daily in two ways: (A) A Pin-10 photo diode was placed over A or B and the output in response to the test flash was observed on an oscilloscope; (B) A Pritchard Spectra Photometer measured the mean luminance of $A$ or $B$ flashing at $1,000 \mathrm{cps}$

During each session the glow modulators were flashed at each of three randomly selected delays, measured from the extinction of $F_{1}$.

\section{Subjects}

Two women, both paid, were used as Os in all the following experiments; a third, a staff member, participated in a few. The Os had at least $20 / 20$ vision, both far and near, in the left eye, as measured with the Armed Forces Vision Tester.
Fig. 3. $d^{\prime}$ measures calculated from the proportions $P(Y / S+N)$ and $P(Y / N)$ shown in Fig. 2 for $O \mathrm{LF}$ and $\mathbf{d}^{\prime}$ measures calculated from a similar $\mathrm{YN}$ experiment for $\mathrm{OHO}$.

\section{RESULTS AND DISCUSSION}

A result typical of the many $\mathrm{YN}$ experiments performed is shown in Fig. 2, which shows the proportions $\mathrm{P}(\mathrm{Y} / \mathrm{S}+\mathrm{N})$, correct detections, and $P(Y / N)$, false alarms, as a function of time relative to the onset of a voluntary saccade. A drop in detection performance, $\mathrm{P}(\mathrm{Y} / \mathrm{S}+\mathrm{N})$ is clearly evident beginning about $100 \mathrm{msec}$ before the saccade begins. ${ }^{3}$ The associated rise in the false alarm rate, $P(Y / N)$, was frequently observed.

Sensitivity measures $\left(d^{\prime}\right)$ were calculated from the proportions given in Fig. 2 and are shown in Fig. 3 along with sensitivity measures for another $O$ in the same experiment. These measures show a progressive decrement in visual sensitivity with minimum sensitivity occurring slightly before the onset of the saccade. Similar data are given in Fig. 4, where $d^{\prime}$ measures calculated from the proportions of correct responses in 2AFC experiment for all three Os are shown. The data of Figs. 3 and 4 demonstrate a saccade-associated decrease in visual sensitivity when changes of the Os' criterion can presumably have no effect. From Figs. 3 and 4 it can be estimated that the attenuation is approximately $0.5 \mathrm{log}$ unit, the value reported by Volkmann (1962), although the attenuation for $O \mathrm{LF}$ is in both cases greater than $1 \log$ unit, approximately the value reported by Zuber \& Stark (1966). It should also be noted that our data suggest that the decrement in visual sensitivity may begin as much as $150-200 \mathrm{msec}$ prior to the onset of the saccade.

The present results also indicate that the $O$ 's criterion may change significantly when judging if flashes have occurred in the temporal vicinity of a voluntary saccade. Although we cannot generalize from our YN experiments, in which blank trials and feedback were used, to previous experiments, which presumably placed no constraints on the O's criterion, we can say that the shifts we have observed tend to attenuate the relation between changes in visual sensitivity and changes in detection performance. ${ }^{4}$ For the precision that would seem to be necessary in the study of saccadic suppression, then, it is suggested that either the behavior of the $O$ 's criterion be measured in conjunction with detection performance, or that criterion-free measures such as those that can be obtained from $2 \mathrm{AFC}$ experiments be used in future parametric studies of the phenomenon. 


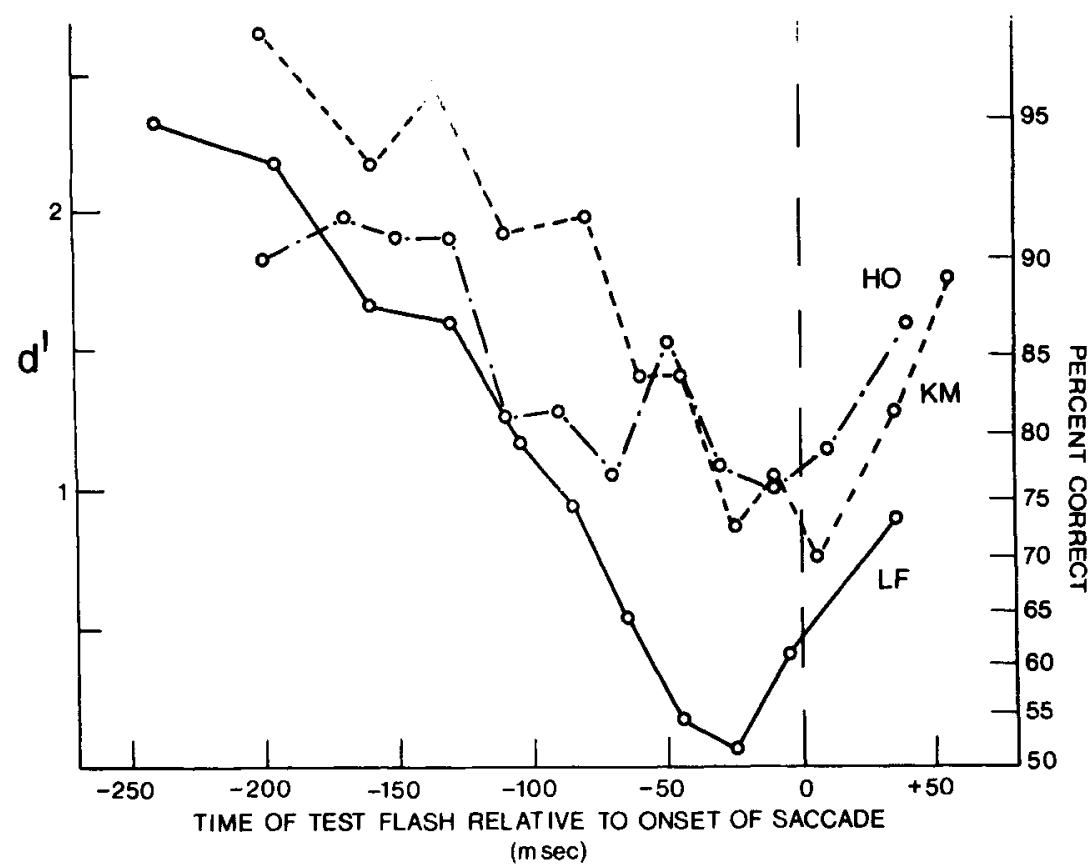

REFERENCES

ENGEL, G. R., \& HOWAT, M. R. The glow modulator as a source for rectangular light flashes. Vision Research, 1965, 6, 479-481.

LATOUR, P. L. Visual threshold durmg eye movements. Vision Research, 1962, 2, 261-262.

LATOUR, P. L. Cortical control of eye movements. Soesterberg, Holland: Institute for Perception, RVO-TNO, 1966.

MATIN, L. Use of glow modulator tube for visual research. American Joumal of Psychology, $1964,77,650-651$.

RICHARDS, W. Saccadic suppression. Joumal of the Optical Society of America, 1969, 59, 617-623.
Fig. 4. $d^{\prime}$ as a function of time relative to the onset of a voluntary saccade for three Os in a $2 \mathrm{AFC}$ experiment. There are approximately 100 observations for each data point.

VOLKMANN, F. C. Vision during voluntary saccadic eye movements. Journal of the Optical Society of America, 1962, 52, 571-578.

VOLKMANN, F. C., SCHICK, A. M. L., \& RIGGS, L. A. Time course of visual inhibition during voluntary saccades. Journal of the Optical Society of America, 1968, 58, 562-569.

ZUBER, B. L., \& STARK, L. Saccadic suppression: Elevation of visual threshold associated with saccadic eye movements Experimental Neurology, 1966, 46, 65-79.

\section{NOTES}

1. DRET Research Paper No. 719.

2. Address: Defence Research Establishment Toronto, P. O. Box 2000, Downsview, Ontario, Canada.

3. Since the responses to flashes occurring after the initiation of the saccade are a function of systematic change in retinal location as well as any general retum of sensitivity following maximum suppression, we have confined our discussion to the data which precede the saccade.

4. The drop in percentage correct in the present YN experiments, generally from about 90 to 50 , is less than usually reported, generally from about 90 to 10 or less. It is possible that these large changes reported by other workers may, in fact, be partly due to upward shifts of the Os' criteria. 